\title{
Polarizable Drude Model with s-type Gaussian or Slater Charge Density for General Molecular Mechanics Force Fields
}

\author{
Mohammad Mehdi Ghahremanpour, ${ }^{\dagger}$ Paul J. van Maaren,${ }^{\dagger}$ Carl \\ Caleman,, Geoffrey R. Hutchison, $₫$ and David van der Spoel ${ }^{*, \dagger}$ \\ $\dagger$ †Upsala Center for Computational Chemistry, Department of Cell and Molecular Biology, \\ Uppsala University, Husargatan 3, Box 596, SE-75124 Uppsala, Sweden \\ $\ddagger$ Department of Physics and Astronomy, Uppsala University, Box 516, SE-75120 Uppsala, \\ Sweden and Center for Free-Electron Laser Science, Deutsches Elektronen-Synchrotron, \\ DE-22607 Hamburg, Germany \\ \Department of Chemistry, University of Pittsburgh, Pittsburgh Pennsylvania 15260, United \\ States \\ E-mail: david.vanderspoel@icm.uu.se
}

\begin{abstract}
Gas phase electric properties of molecules can be computed routinely using wave function methods or the density functional theory (DFT). However, these methods remain computationally expensive for high-throughput screening of the vast chemical space of virtual compounds. Therefore, empirical force fields are a more practical choice in many cases, particularly since force field methods allow routinely predicting of physicochemical properties in the condensed phases. This work presents Drude polarizable models, to increase the physical realism in empirical force fields, where the core particle is treated as a point charge and the
\end{abstract}


Drude particle is treated either as a $1 s$-Gaussian or a $n s$-Slater $(n=1,2,3)$ charge density. Systematic parametrization to large high quality quantum chemistry data obtained from the open access Alexandria Library (https://doi.org/10.5281/zenodo.1004711) ensures the transferability of these parameters. The dipole moments and isotropic polarizabilities of the isolated molecules predicted by the proposed Drude models are in agreement with experiment with accuracy similar to DFT calculations at the B3LYP/aug-cc-pVTZ level of theory. The results show that the inclusion of explicit polarization into the models reduces the root mean square deviation with respect to DFT calculations of the predicted dipole moments of 152 dimers and clusters by more than $50 \%$. Finally, we show that the accuracy of the electrostatic interaction energy of the water dimers can be improved systematically by the introduction of polarizable smeared charges as a model for charge penetration.

Keywords: Alexandria Force Field, GROMACS, Drude Oscillators, Smeared Charge Models, Generalized Amber Force Field

\footnotetext{
Abbreviations: DFT: Density Functional Theory, PC: Point Charge, PPC: Polarizable Point Charge, PGC: Polarizable Gaussian Charge, PSC: Polarizable Slater Charge
}

\section{INTRODUCTION}

Prediction of physicochemical properties of arbitrary compounds is central in molecular engineering. Electrostatic interactions along with charge polarization and the possibility of charge penetration, overlapping of electron densities at close distances, are crucial to understand the electric properties of molecules. ${ }^{1,2}$ The explicit inclusion of polarization into empirical force fields through polarizable point charges has improved the classical description of molecular electrostatics. ${ }^{1,3-7}$ However, there is an inherent problem with the point charge model, which is the singularity at short distance. This is known to cause the so called polarization catastrophe at short distances, ${ }^{8}$ particularly in condensed-phase simulations. A remedy for the polarization catastrophe was devised by Thole who proposed damping the electrostatic forces at close interatomic distances..$^{9,10}$ This is needed because the Coulomb potential varies rapidly as a function of distance for point 
charges, especially at short distances, which makes it necessary to perform molecular dynamics (MD) simulations using very short time steps to conserve energy. ${ }^{11}$ The point charge model also fails to account for charge penetration ${ }^{12}$ and therefore, more elaborate functional forms may be needed to describe all electrostatic effects in MD simulations.

Hall et al. modeled atomic partial charges by spherical Gaussian functions in molecular mechanics (MM) calculations already by $1986 .{ }^{13,14}$ They showed that by using one point charge combined with at least one diffuse Gaussian function on each atom a realistic molecular electrostatic potential (MEP) can be obtained, in agreement with quantum mechanical (QM) calculations. ${ }^{13}$ Later, Rappé and Goddard modeled partial atomic charges by normalized valence Slater $s$-orbitals to shield the interaction between charge densities in MD simulations. ${ }^{15}$ Despite these early attempts, treating partial atomic charges as smeared (screened) charges in empirical force fields has until recently not received much consideration because of both limited computational resources and due to most force fields being parameterized for point charges. However, there is no fundamental problem to solve the Coulomb integral for smeared charges in molecular mechanics, in particular for Gaussian distributions. ${ }^{16}$ Smeared charges have been used, for instance, to improve the quality of the fit to the electrostatic potential on a grid around a carbon monoxide and water dimers compared to the point multipole expansion. ${ }^{17}$

The inclusion of charge penetration effects through smeared charge models is a natural step to higher accuracy in molecular mechanics force fields. It was shown, for instance, to increase the accuracy of multipole electrostatic energies used to calculate the interaction energy of homoand hetero- dimers. ${ }^{12}$ In another example, a model with an explicit energy term for charge penetration was developed to reproduce the experimental interaction energies in $\mathrm{CO}_{2}$ clusters. ${ }^{18}$ The contribution of charge penetration to the electrostatic energy of a system is likely larger in the condensed phases than the gas phase, since short distances are sampled more, particularly at room- or higher temperatures. Accounting for the charge penetration effect in MM force fields also yields more accurate interactions between QM orbitals and MM partial charges in electronically embedded QM/MM 2 and MM calculations with screened charges produce more accurate electrostatics 
than QM/MM calculations with point charges. ${ }^{19}$ Polarizable smeared charges have been developed based on the charge-on-spring approach to model carbon dioxide, ${ }^{20}$ alkali-halides ${ }^{21}$ and water. ${ }^{22-24}$ An induced dipole polarization model has been developed for interacting Gaussian $s$ and $p$-orbitals ${ }^{25}$ and compared to the damped Thole model. ${ }^{9}$ It was found that the Gaussian model yields more accurate molecular isotropic polarizabilities than the Thole model. ${ }^{25}$ These studies altogether show advantages of screened charges over the point charge model, however, they have only been applied to either specific compounds or small sets of molecules. Therefore, parametrization of transferable spherical charge densities is addressed here.

The objective of this paper is to provide a systematic platform to derive transferable exponents of polarizable atom-centered $s$-type Gaussian and Slater density functions as well as atomic polarizabilities for use in empirical force fields. These parameters are derived based on the Alexandria library of optimized molecular geometries and electronic properties. ${ }^{26,27}$ Uncertainties in the orbital exponents and atomic polarizabilities are determined using the Bayesian formalism and the bootstrapping regression model, respectively. This will allow to quantify uncertainties due to force field parameters in predictions from e.g. MD simulations. ${ }^{28}$ In a previous study, Slater charge densities were directly fitted to a reference molecular electron density. ${ }^{29}$ Therein, the Minimal Basis Iterative Stockholder (MBIS) was proposed to circumvent the problems of the partitioning of an electron density into pro-atom densities. ${ }^{29}$ In other work, electrostatic interaction energies were used for fitting parameters of Slater-type functions. ${ }^{12}$ In the present work, the Gaussian and Slater functions are parametrized to reproduce molecular dipole moments and electrostatic potentials. The dipole moment is the first moment of the electron density and the molecular electrostatic potential is linked to the electron density through the Poisson equation. ${ }^{30} \mathrm{MEP}$ is also an informative quantity in itself that is related, for instance, to the reactive behavior of a molecule. ${ }^{13,30}$ Therefore, we have implemented an ESP fitting algorithm, ${ }^{31}$ despite it's known shortcomings, ${ }^{32}$ in combination with a number of other tools in order to parameterize polarizable Gaussian- and Slater-type charge models. 


\section{THEORY}

\section{Slater Charge Density}

The wave function of the outermost spherical Slater orbital is given by

$$
\psi_{n}(\mathbf{r})=\sqrt{\frac{(2 \zeta)^{2 n+1}}{4 \pi(2 n) !}} \mathbf{r}^{n-1} \mathrm{e}^{-\zeta \mathbf{r}}
$$

where $n$ is the highest principal quantum number of the element and $\zeta$ is the orbital exponent determining the diffuseness of the charge density. The distribution of atomic partial charges in MM force fields can be described by the charge density, which is the square of the wave function (Eqn. 1). Using this, the Coulomb integral can be written as: ${ }^{33,34}$

$$
J_{i j}(\mathbf{r}) \sim \iint\left|\psi_{n}\left(\mathbf{r}_{\mathbf{i}}\right)\right|^{2} \frac{q_{i} q_{j}}{\left|\mathbf{r}_{\mathbf{i}}-\mathbf{r}_{\mathbf{j}}\right|}\left|\psi_{m}\left(\mathbf{r}_{\mathbf{j}}\right)\right|^{2} d \mathbf{r}_{\mathbf{i}} d \mathbf{r}_{\mathbf{j}}
$$

where $\psi_{n}$ and $\psi_{m}$ are the Slater wave functions of atoms $i$ and $j$ with quantum numbers $n$ and $m$ and with partial charges $q_{i}$ and $q_{j}$, respectively. Eqn. 2 has a finite limit as $r \rightarrow 0$, hence, it is well behaved at small values of $r$ and it monotonously decreases for all $n$. Several methods ${ }^{17,35}$ have been developed to evaluate Eqn. 2. Hentschke gives the following analytical solution: ${ }^{34}$

$$
\begin{aligned}
J_{i j}(\mathbf{r})= & \frac{1}{4 \pi \epsilon_{0}} \frac{q_{i} q_{j}}{\left|\mathbf{r}_{\mathbf{i}}-\mathbf{r}_{\mathbf{j}}\right|} \frac{4 \zeta_{i}^{2 n+1} \zeta_{j}^{2 m+1}}{(2 n) !(2 m) !} \frac{\partial^{2 n-2} \partial^{2 m-2}}{\partial \zeta_{i}^{2 n-2} \partial \zeta_{j}^{2 m-2}} \frac{1}{\zeta_{i}^{3} \zeta_{j}^{3}} \\
& {\left[1-\frac{\left(3 \zeta_{i}^{2}-\zeta_{j}^{2}\right) \zeta_{j}^{4}}{\left(\zeta_{i}-\zeta_{j}\right)^{3}\left(\zeta_{i}+\zeta_{j}\right)^{3}} e^{-2 \zeta_{i} r_{i j}}-\frac{\left(\zeta_{i}^{2}-3 \zeta_{j}^{2}\right) \zeta_{i}^{4}}{\left(\zeta_{i}-\zeta_{j}\right)^{3}\left(\zeta_{i}+\zeta_{j}\right)^{3}} e^{-2 \zeta_{j} r_{i j}}\right.} \\
& \left.-\frac{\zeta_{i} \zeta_{j}^{4}}{\left(\zeta_{i}-\zeta_{j}\right)^{2}\left(\zeta_{i}+\zeta_{j}\right)^{2}} r_{i j} e^{-2 \zeta_{i} r_{i j}}-\frac{\zeta_{i}^{4} \zeta_{j}}{\left(\zeta_{i}-\zeta_{j}\right)^{2}\left(\zeta_{i}+\zeta_{j}\right)^{2}} r_{i j} e^{-2 \zeta_{j} r_{i j}}\right]
\end{aligned}
$$

Eqn. 3 was implemented in a Mathematica program from which $\mathrm{C}++$ code was generated for the analytical computation of $J_{i j}$ and its analytical derivatives with respect to $r$, which are necessary for computing forces. Due to the nature of Eqn. 3, there are many terms with large powers, 
particularly for $n>3$. Thus, the equations have to be implemented using the arbitrary precision arithmetic library "Class Library for Numbers" (http://www.ginac.de/CLN/) to avoid numerical instabilities. However, an arbitrary high precision significantly increases the computational cost to analytically solve Eqn. 3, which will lead to a poor exploration of the parameter space when optimizing the Slater exponents. Therefore, we used a Slater 3s orbital rather than the valence Slater $s$-orbital for the elements of the $4^{t h}$ and the $5^{\text {th }}$ rows of the periodic table. This approximation allows using a double precision implementation to solve Eqn. 3 without numerical instabilities.

\section{Gaussian Charge Density}

Gaussian-type orbitals have the form:

$$
\psi(\mathbf{r})=\left(\frac{2 \xi}{\pi}\right)^{\frac{3}{4}} e^{-\xi \mathbf{r}^{2}}
$$

where $\xi$ determines the diffuseness of the orbital. The probability (electron) density function is the square of Eqn. 4, which is used here to describe the distribution of atomic partial charges in order to reduce the computational complexity compared to the Slater wave function:

$$
\rho(\mathbf{r})=\left(\frac{2 \xi}{\pi}\right)^{\frac{3}{2}} e^{-2 \xi \mathbf{r}^{2}}
$$

Note that the unit of the $\xi$ is $\mathrm{nm}^{-2}$ while the unit of $\zeta$ in Eqn. 1 is $\mathrm{nm}^{-1}$. In order to make the units comparable, Eqn. 5 is rearranged as: ${ }^{36}$

$$
\rho(\mathbf{r})=\left(\frac{\beta^{2}}{\pi}\right)^{\frac{3}{2}} e^{-\beta^{2} \mathbf{r}^{2}}
$$

where $\beta=\sqrt{2 \xi}$. The solution of the Coulomb integral of two interacting $1 s$-Gaussian charge densities can then be written as:

$$
J_{i j}(\mathbf{r})=\frac{1}{4 \pi \epsilon_{0}} \frac{q_{i} q_{j}}{\left|\mathbf{r}_{\mathbf{i}}-\mathbf{r}_{\mathbf{j}}\right|} \operatorname{erf}\left(\beta_{i j} r_{i j}\right)
$$


where

$$
\beta_{i j}=\frac{\beta_{i} \beta_{j}}{\sqrt{\beta_{i}^{2}+\beta_{j}^{2}}} .
$$

Note that for $i=j, \beta_{i i}=\beta_{i} / \sqrt{2}$ and that the Gaussian interaction function is well behaved as $r \rightarrow 0$.

\section{Polarizability}

The classical Drude oscillator formalism is used to explicitly take polarizability into account in the Alexandria force field that is under development. Drude oscillators have extensively been explained elsewhere ${ }^{3,4,37-44}$ and have been compared to other approaches for the inclusion of electronic polarizability into force fields. ${ }^{45-47}$ In this formalism, an atom is represented as a two-particle system - a core particle that is connected to a Drude particle by a harmonic spring. A massless Drude particle is often referred to as a shell ${ }^{3,4,10,37,48}$ and is not necessarily located at the core, it can also be used, for instance, to model bonding orbitals. ${ }^{3}$ The charge of the atom $\left(q^{a}\right)$ is the sum of the positive charge on the core $\left(q^{c}\right)$ and the charge on the shell $\left(q^{s}\right)$ which is chosen to be negative to represent the electron cloud. The atomic self-polarization energy $\left(U_{\text {self }}^{a}\right)$ is expressed as the harmonic energy between the core and the shell:

$$
U_{\text {self }}^{a}=\frac{1}{2} k d^{2}
$$

where $d$ is the core-shell distance under the influence of the electric field induced by other atoms and $k$ is the force constant of the spring defined as

$$
k=\frac{q^{s^{2}}}{\alpha^{a}}
$$

where $\alpha^{a}$ is the atomic polarizability. $k$ is sometimes set to the same constant for all core-shell pairs rand $q^{s}$ is then tuned to achieve an appropriate $\alpha^{a}{ }^{49}$ In this scenario, the atomic polarizability is defined by the partial charge of the shell particle, which is not a physical observable. Therefore, we prefer to derive $\alpha^{a}$ from experimental molecular polarizability because it is a physical observ- 
able. Based on Lorentz's additive law of optical refractivity, ${ }^{50}$ the molecular mean polarizability $\left(\bar{\alpha}^{m}\right)$ can be approximated by the sum of the atomic polarizabilities: ${ }^{51}$

$$
\bar{\alpha}^{m}=\sum_{i}^{N} \alpha_{i}^{a}
$$

The additive law has been tested by measuring the refractive index of proteins that is indeed equal to the sum of amino-acid polarizabilities. ${ }^{52}$ The electronic polarizabilities of the nucleic acid bases also follow the atomic polarizability additive law. ${ }^{53}$ Bosque and Sales derived an empirical formula relating molecular polarizability at $589 \mathrm{~nm}$ frequency to the atomic polarizability as following:

$$
\bar{\alpha}^{m}=0.32+\sum_{i}^{N} \alpha_{i}^{a}
$$

where $\alpha_{i}^{a}$ was derived for the elements ${ }^{54}$ by applying the law of additivity to decompose molecular polarizability into atomic polarizabilities. It should be noted that it was pointed outed already 100 years ago that the simple additivity law may not work in all cases. ${ }^{50}$ It may, for instance, break down on conjugated molecules with multiple double bonds, which have also been shown to be challenging for high level quantum mechanical methods. ${ }^{55}$

Having polarizable atoms, the molecular polarizability tensor $\left(\boldsymbol{\alpha}^{m}\right)$ can be calculated. This yields the proportionality between the strength of an applied electric field and the change in the electric dipole moment vector induced by the electric field:

$$
\Delta \boldsymbol{\mu}=\boldsymbol{\alpha}^{m} \boldsymbol{E}
$$

The isotropic molecular polarizability can then be calculated as the average of the trace of the $\boldsymbol{\alpha}^{m}$ tensor as follows: ${ }^{53,56}$

$$
\bar{\alpha}^{m}=\frac{1}{3}\left(\alpha_{x x}^{m}+\alpha_{y y}^{m}+\alpha_{z z}^{m}\right) .
$$


Due to interactions between polarizable atoms, that molecular polarizability obtained from Eqn. 14 is not the same as the additive sum of the atomic polarizabilities (Eqn. 11)

\section{DATABASE}

The Alexandria library has recently been introduced ${ }^{27}$ to facilitate systematic development of force field models. It is a database of quantum-chemical calculations of molecular thermochemistry ${ }^{57}$ as well as molecular optimized geometry, electrostatic potential map, electric moments and the polarizability tensor at the B3LYP/aug-cc-pVTZ level of theory. ${ }^{58-64}$ The library, that can be downloaded from Zenodo, ${ }^{26,27}$ contains metals, inorganic, and organic compounds. Here, we limit ourselves to molecules containing atoms of biological interests $(\mathrm{H}, \mathrm{C}, \mathrm{N}, \mathrm{O}, \mathrm{P}, \mathrm{S})$ and halogens $(\mathrm{F}, \mathrm{Cl}$, $\mathrm{Br}, \mathrm{I})$ that are present in many drug-like molecules.

For testing the charge models derived here, several benchmark sets were used. Molecular homo- and hetero-dimers available from the Binding Energy Database (http://begdb.com) were downloaded in order to examine the methodology more rigorously by predicting dipoles of the dimers and comparing them to DFT calculations. The sets of dimers used are summarized in Table 1. S66 dataset contains dimers of biomolecule-related compounds, ${ }^{65,66} \mathrm{X} 40$ includes dimers

of halogen compounds, ${ }^{67}$ SHBC contains dimers having halogen bonds. ${ }^{68}$ The water dataset consists of water clusters, from 2 to 10 molecules . ${ }^{69}$

Table 1: Overview of clusters used for testing dipoles.

\begin{tabular}{llcl}
\hline Set & Description & Number & Reference \\
\hline SHBC & Halogen bonding & 6 & 68 \\
S66 & Biomolecule-related & 66 & 65,66 \\
X40 & Organic halogen & 40 & 67 \\
Water & Water clusters & 40 & 69 \\
\hline
\end{tabular}




\section{METHODS}

\section{Generation of Atom Types}

In order to derive transferable parameters that can be used in molecular simulations, atom types must be determined in all compounds. For this purpose we used the Open Babel software ${ }^{70}$ that uses SMiles ARbitrary Target Specification (SMARTS) patterns ${ }^{71}$ to generate atom types for the Generalized Amber Force Field. ${ }^{72}$ The atom types generated by Open Babel were compared to results from the Antechamber software, version $16^{73}$ and using this comparison the SMARTS definitions were "debugged". Although Antechamber in general should be considered authoritative, there are some difficult cases where the software is inconsistent with the paper. ${ }^{72}$ For these cases the SMARTS patterns in Open Babel (available in version 2.4.1 or later) were modified to reproduce the published definitions.

\section{ESP-fitting with Drude Models}

The Electrostatic Potential (ESP) fitting algorithm was implemented to work with Drude models. The algorithm fits the atomic partial charges to reproduce the quantum mechanical electrostatic potential at a series of points around the molecule. ${ }^{31,74}$ This boils down to, in principle, a constrained least-squares fitting procedure that can be written in matrix notation: ${ }^{75}$

$$
\left[\begin{array}{l}
J \\
V
\end{array}\right]\left[\begin{array}{l}
q
\end{array}\right]=\left[\begin{array}{l}
u \\
v
\end{array}\right]
$$

Because we have $m$ grid points and $n$ polarizable atoms, $\boldsymbol{J}$ is the $m \times n$ Coulomb matrix whose elements, $J_{i j}$, are computed from the distance between atoms $j$ in the molecule and the grid points $i$ around the molecule $\left(r_{i j}\right)$ using the kernel of Coulomb's law derived in Eqn. 3 or Eqn. 7, or, for point charges $J_{i j}=\frac{1}{4 \pi \epsilon_{0} r_{i j}}$. The vector $\boldsymbol{u}$ stores the quantum mechanical electrostatic potentials at each grid point. $\boldsymbol{V}$ is an $k \times n$ matrix appended to $\boldsymbol{J}$ containing $k$ linear equations to fulfill $k$ 
constraints encoded in vector $\boldsymbol{v}$ that is appended to $\boldsymbol{u}$ on the right-hand side of the equation. The constraints are imposed to ensure that the charges on symmetrically equivalent atoms (e.g. methyl groups) are equal and that the sum of partial charges equals the total charge of the molecule $\left(Q^{T}\right)$. For instance, in the example below, the charges on atoms 1 and 3 are constrained to be equal to the charge on atoms 2 , and $n$, respectively.

$$
\left[\begin{array}{ccccc}
J_{11}^{c} & J_{12}^{c} & J_{13}^{c} & \cdots & J_{1 n}^{c} \\
J_{21}^{c} & J_{22}^{c} & J_{23}^{c} & \cdots & J_{2 n}^{c} \\
\vdots & \vdots & \vdots & \ddots & \vdots \\
J_{m 1}^{c} & J_{m 2}^{c} & J_{m 3}^{c} & \cdots & J_{m n}^{c} \\
V_{11}^{p} & V_{12}^{p} & V_{13}^{p} & \cdots & V_{1 n}^{p} \\
V_{21}^{p} & -V_{22}^{p} & 0 & \cdots & 0 \\
\vdots & \vdots & \vdots & \ddots & \vdots \\
0 & 0 & V_{k 3}^{p} & \cdots & -V_{k n}^{p}
\end{array}\right]\left[\begin{array}{c}
q_{1}^{c} \\
q_{2}^{c} \\
q_{3}^{c} \\
\vdots \\
q_{n}^{c}
\end{array}\right]=\left[\begin{array}{c}
\phi_{1}-J_{1}^{s} \\
\phi_{2}-J_{2}^{s} \\
\vdots \\
\phi_{m}-J_{m}^{s} \\
Q^{T}-\sum_{j}^{n} q_{j}^{s} \\
0 \\
\vdots \\
0
\end{array}\right]
$$

In Eqn. 16, $\phi_{i}$ is the quantum mechanical electrostatic potential on the grid point $i, J_{i j}^{c} q_{j}^{c}$ is the electrostatic potential on the grid point $i$ produced by the charge $q_{j}^{c}$ on the core of atom $j$, and $J_{i}^{s}$ is the electrostatic potential on grid point $i$ produced by all the shell particles. $V_{i j}^{p}$ is a weighting factor penalizing the fit to ensure that the constraints encoded in vector $v$ are satisfied. To simplify the fitting procedure we only varied $q_{j}^{c}$, which means that the contributions of the shells are constant terms in the linear equations of matrix $\boldsymbol{J}$; hence, they were moved to the vector $\boldsymbol{u}$ on the right-hand side of Eqn. 15. The value of $q^{s}$ was set to -1 for hydrogen atoms and to -2 for the other supported elements. Eqn. 16 can be abbreviated as:

$$
A q=b
$$


and it can be solved by the singular value decomposition (SVD) algorithm. We then compute the goodness of fit from:

$$
\chi^{2}=(\boldsymbol{A q}-\boldsymbol{b})^{2}
$$

Because the shell particles contribute to the right-hand side of Eqn. 17, the charge generating algorithm must be combined with the optimization of the polarizable shell positions. This can be solved iteratively (Fig. 1).

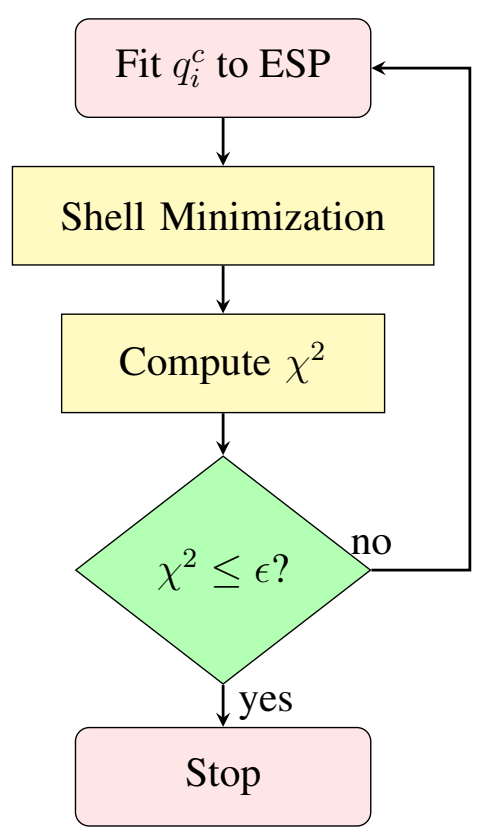

Figure 1: Flowchart of the self-consistent algorithm for fitting electrostatic potentials with a Drude model. Shell minimization indicates performing an energy minimization of the shell particle positions in the field of the fixed core particles in order to ensure that the force on every shell particle is zero at every iteration. Minimization of the position of the shell particles is carried out by software from the GROMACS package. ${ }^{76}$

\section{Fitting Atomic Polarizabilities}

The SVD algorithm was applied to derive atomic polarizabilities from experimental molecular polarizabilities based on Eqn. 11. A more detailed description of the fitting procedure follows from the matrix equation: 


$$
\left[\begin{array}{ccccc}
a_{1, h a} & a_{1, h p} & a_{1, c 1} & \cdots & a_{1, i} \\
a_{2, h a} & a_{2, h p} & a_{2, c 1} & \cdots & a_{2, i} \\
\vdots & \vdots & \vdots & \ddots & \vdots \\
\vdots & \vdots & \vdots & \ddots & \vdots \\
a_{k, h a} & a_{k, h p} & a_{k, c 1} & \cdots & a_{k, i}
\end{array}\right]\left[\begin{array}{c}
\alpha_{h a}^{a} \\
\alpha_{h p}^{a} \\
\vdots \\
\alpha_{i}^{a}
\end{array}\right]=\left[\begin{array}{c}
\bar{\alpha}_{1}^{m} \\
\bar{\alpha}_{2}^{m} \\
\vdots \\
\vdots \\
\bar{\alpha}_{k}^{m}
\end{array}\right]
$$

where $a_{i, h a}$ is the occurrence of polarizability type $h a$ in molecule $i, \alpha_{h p}^{a}$ is the polarizability value of $h p$ and $\bar{\alpha}_{i}^{m}$ is the isotropic polarizability of molecule $i$. Here, the $\alpha_{x}^{a}$ are derived for the GAFF atom types. ${ }^{72}$ Similar GAFF atom types were lumped together to reduce the number of parameters. In doing so, $64 \mathrm{GAFF}$ atom types for $\mathrm{H}, \mathrm{C}, \mathrm{N}, \mathrm{O}, \mathrm{P}, \mathrm{S}$ and halogens $(\mathrm{F}, \mathrm{Cl}, \mathrm{Br}, \mathrm{I})$ were combined to 17 groups, each of which represents a "polarizability type" in the Alexandria force field (Table 2).

Table 2: Alexandria polarizability types.

\begin{tabular}{ll}
\hline Polarizability type & GAFF atom types ${ }^{72}$ \\
\hline ha & h1, h2, h3, h4, h5, ha, hc \\
hp & hn, ho, hp, hs \\
c1 & c1 \\
c2 & c, c2, cc, cd, cg, cp, cq, ce, cf, cu, cv, cz \\
ca & ca \\
c3 & c3, cx, cy \\
n & n, n1, n2, n3, n4, nc, nd, ne, nh \\
na & na, nb \\
no & no \\
o & o, oh, os \\
f & f \\
p2 & p2, p3, pb, pc, pe, px, py \\
p4 & p4, p5 \\
s & s, s4, s6, sh, ss, sx, sy \\
cl & cl \\
br & br \\
i & i \\
\hline
\end{tabular}

A bootstrapping regression model ${ }^{77}$ was used to calculate the uncertainty in $\alpha^{a}$ by generating 1000 bootstrapped samples. For each bootstrapped sample, $k$ molecules were selected randomly with replacement from the original sample to solve Eqn. 19. A histogram of bootstrap means was 
then created for each $\alpha^{a}$. This histogram provided an estimate of the optimum value of $\alpha^{a}$ and how much it varies across samples.

\section{Parametrizing s-type Charge Densities}

The statistical machine learning approach we used to parametrize the Gaussian and Slater density functions is inspired by the Bayesian formalism which allows to propagate the uncertainty in the model coming from the static model parameters. A probability distribution was defined in the parameter space given the choice of model (M) and data (D) as follows: ${ }^{78,79}$

$$
p(\Theta \mid M, D) \propto \exp \left[-\frac{E(\Theta)}{T}\right]
$$

where $\Theta$ denotes the vector of parameters, $T$ is the effective "temperature" that is in principle the weighting of different parameter sets, and $E(\Theta)$ is the loss function:

$$
E(\Theta)=\Omega \boldsymbol{X}^{2}+\Lambda
$$

where $\boldsymbol{X}^{2}$ is the vector of residuals in the least squares form weighted by vector $\Omega$. Here, the components of $\boldsymbol{X}^{2}$ were the residual of the components of the molecular dipole moments $\left(\chi_{\mu}^{2}\right)$ and of the electrostatic potentials $\left(\chi_{\phi}^{2}\right)$ :

$$
\begin{aligned}
\chi_{\mu}^{2} & =\frac{1}{3 N} \sum_{i}^{N} \sum_{j}^{3}\left(\mu_{i j}^{M M}-\mu_{i j}^{Q M}\right)^{2} \\
\chi_{\phi}^{2} & =\frac{1}{N K} \sum_{i}^{N} \sum_{j}^{K}\left(\phi_{i j}^{M M}-\phi_{i j}^{Q M}\right)^{2}
\end{aligned}
$$

where $N$ is the number of molecules and $K$ is the number of electrostatic potential grids (note that $N \ll K)$. Different $\Omega$ vectors were used at different stages of the optimization to improve the performance of the fitting. For the final step of the optimization, $\Omega_{\mu}$ was set to 100 and $\Omega_{\phi}$ was set to $1 . \Lambda$ in Eqn. 21 is a $l_{2}$-norm regularizer that discourages overfitting. It restrains the optimization 
to search in a region of the parameter space confined by hyperparameters $L$ and $U$ as the lower and upper bounds, respectively. It is given by:

$$
\Lambda=\frac{1}{2}\left[\sum_{i}\left(\theta_{i}-L\right)^{2} H_{L}\left(-\theta_{i}\right)+\sum_{i}\left(\theta_{i}-U\right)^{2} H_{U}\left(\theta_{i}\right)\right]
$$

where $H$ denotes the Heaviside function. The hyperparameters $L$ and $U$ are chosen by scrutiny of our initial guess of the values of parameter $\zeta$ that were optimized previously, ${ }^{19}$ except Iodine. Initial values for Iodine were guessed based on the value for Bromine. ${ }^{19}$ Similarly, different sets of initial values were randomly chosen for $\beta$. After the trust region was found for each parameter, a box-constrained optimization was performed to fine-tune each parameter in its trust region.

A Metropolis-Hastings (MH) algorithm combined with Simulated Annealing (SA) was developed in-house to explore the posterior distribution of parameters by minimizing the loss function under the conditions explained above. The optimization protocol was applied to 10 replicas randomly generated as the training sets. Each replica contained about 500 compounds of the Alexandria library. The optimum value and the uncertainty for each parameter is then respectively obtained by calculating the average and standard deviation over the replicas.

\section{Computational Details}

Dipole moments of the dimers at the B3LYP/aug-cc-pVTZ level of theory were calculated by the Gaussian package ${ }^{80}$ (version 16) and have been described earlier. ${ }^{26,27}$ The Psi4 open source package $^{81}$ was used to calculate the electrostatic interaction energy of the water dimer at different separation distances based on the Symmetry-Adapted Perturbation Theory (SAPT). ${ }^{82}$ These calculations were done at the SAPT2+/aug-cc-pVTZ level. ${ }^{83}$ The dipole moments and the electrostatic interaction energy of the dimers using the Alexandria charge models were computed with the GROMACS package ${ }^{76}$ (version 2018). Table look-up was used to interpolate electrostatic interactions in GROMACS with a table spacing of $0.002 \mathrm{~nm} .^{76}$ The interaction of atom $\mathbf{i}$ with atoms $\mathbf{i}+\mathbf{1}$ and $\mathbf{i}+\mathbf{2}$, that are covalently bonded were excluded. The 1-4 intramolecular interactions 
were included but not scaled.

\section{RESULTS}

Previous studies typically examined the accuracy of force fields in reproducing just the magnitude of the dipole vector and the isotropic polarizability of molecules, ${ }^{6,84,85}$ in part due to lack of experimental data. Although there have been studies using the dipole moment and the polarizability along the molecular axes for specific compounds like water, ${ }^{4}$ there does not seem to exist any large dataset of components of the dipole vector or the polarizability tensor. No large-scale data set of the molecular quadrupole tensors appears to exist either. Therefore, we have assessed the transferability of the atomic polarizabilities and the orbital exponents by benchmarking not only the total dipole moment and the isotropic polarizability but also the components of the dipole vector and the diagonal elements of the polarizability and quadrupole tensors to the density functional theory calculations from the Alexandria library. ${ }^{26,27}$ Then, the performance of the charge models was evaluated by computing the dipole vector of homo- and hetero-dimers as well as water clusters and comparing the results to DFT calculations. Finally, the charge models were validated by computing the electrostatic interaction energy of a water dimer at different distances along the hydrogen bond-breaking coordinate and comparing the energies to quantum chemistry results.

\section{Optimized Parameters}

Here, we present the optimum value and the uncertainty in the polarizability and the exponent of the Gaussian and Slater charge densities obtained for the Alexandria polarizability types (Table 2). The water oxygen (ow) and hydrogen $(h w)$, shown in Tables 3 and 4, are defined separately in this work for both atom- and polarizability type.

Table 3 lists the optimal value of atomic polarizabilities and compares them to other studies.

The initial polarizability value of $h w$ and $o w$ was taken from a previous study. ${ }^{25}$ Some experimenting led to the finding that a $1 \%$ increase in the ow polarizability yielded different charges in the 
ESP fitting that, in combination, yielded very accurate electrostatic energies and also reproduced the experimental isotropic polarizability of $1.44\left(\AA^{3}\right)$ for water. ${ }^{86}$ Although manual tinkering with the parameters is not reproducible per se, there are uncertainties in the ESP as well, because it depends on the level of theory chosen and, in addition, there is no statistics for the $o w$ and $h w$ atom types since they occur in water only. Other polarizability values were obtained from the decomposition procedure explained above. The Alexandria polarizability values are in good agreement with the atomic hybrid polarizabilities (Ahp) which are the refined values of the atomic hybrid components (Ahc). ${ }^{87}$ The Ahp values depend on both the identity and the hybridization state of a particular atom, which is similar to the concept of force field atom types. On the other hand, the Alexandria polarizabilities differ slightly from those determined by Bosque and Sales for the elements. ${ }^{54}$ The results suggest that the polarizability of the elements depends on the hybridization state and the chemical environment. For instance, the hypervalent phosphorous $(p 4)$ is less polarizable than its $\mathrm{sp}^{2}$ and $\mathrm{sp}^{3}$ hybridizations shown as $p 2$ in Table 3. Bootstrapped distributions are plotted in Fig. 2 showing the uncertainty in the optimal value of atomic polarizabilities. The obtained distributions are relatively unimodal for all polarizability types listed in Table 3, except for $p 2$, which is slightly left-skewed. The small standard deviations of the distributions imply a low uncertainty in the atomic polarizabilities, due to the number and the type of molecules for which experimental polarizability was accessible.

Table 3: Atomic polarizability $\left(\AA^{3}\right)$ obtained from the decomposition of the experimental isotropic molecular polarizability. $N$ is the number of experimental datapoints used. The columns Ahc and Ahp contain atomic hybrid components ${ }^{88}$ and atomic hybrid polarizabilites, ${ }^{51,87}$ respectively. The column BS contains the polarizabilities of Bosque and Sales. ${ }^{54}$ The uncertainty, $\sigma$, in the Alexandria polarizability values are computed by Bootstrapping with 1000 interations.

\begin{tabular}{lccccc}
\hline Polarizability Type & $N$ & \multicolumn{5}{c}{ Polarizability } \\
& & Alexandria $(\sigma)$ & Ahc & Ahp & BS \\
\hline ha & 1110 & $0.384(0.012)$ & 0.392 & 0.387 & 0.170 \\
hp & 252 & $0.432(0.014)$ & 0.392 & 0.387 & 0.170 \\
hw & 1 & $0.376(-)$ & - & - & 0.170 \\
c1 & 69 & $1.459(0.015)$ & 1.294 & 1.283 & 1.510 \\
ca & 205 & $1.411(0.014)$ & - & - & 1.510 \\
c2 & 464 & $1.417(0.018)$ & 1.369 & 1.352 & 1.510 \\
c3 & 1036 & $1.074(0.027)$ & 1.116 & 1.061 & 1.510 \\
\hline
\end{tabular}




\begin{tabular}{lccccc}
\hline Polarizability Type & $N$ & \multicolumn{5}{c}{ Polarizability } \\
& & Alexandria $(\sigma)$ & Ahc & Ahp & BS \\
\hline na & 28 & $0.863(0.078)$ & - & - & 1.050 \\
n & 147 & $0.928(0.023)$ & 1.077 & 0.964 & 1.050 \\
no & 14 & $1.657(0.063)$ & - & - & 1.050 \\
ow & 1 & $0.689(-)$ & - & - & 0.570 \\
o & 420 & $0.565(0.010)$ & 0.780 & 0.637 & 0.570 \\
f & 68 & $0.415(0.016)$ & 0.527 & 0.296 & 0.220 \\
p4 & 7 & $2.214(0.060)$ & - & - & 2.480 \\
p2 & 8 & $3.044(0.314)$ & - & - & 2.480 \\
s & 71 & $2.932(0.041)$ & 3.056 & 3.000 & 2.990 \\
cl & 105 & $2.307(0.013)$ & 2.357 & 2.315 & 2.160 \\
br & 42 & $3.441(0.023)$ & 3.541 & 3.013 & 3.290 \\
i & 17 & $5.459(0.090)$ & 5.573 & 5.415 & 5.450 \\
\hline
\end{tabular}

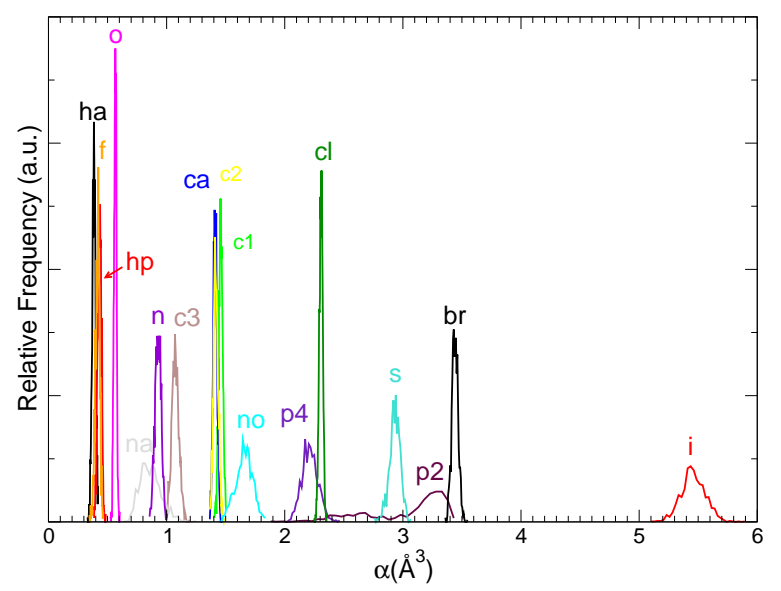

Figure 2: Distribution of atomic polarizabilities. Each distribution was obtained from bootstrapping with 1000 iterations. At each iteration, a sample of experimental molecular polarizabilities was built randomly with replacement to perform singular value decomposition.

The optimized exponents of Gaussian and Slater density functions are given in Table 4. In these models, the charge on the core is treated as a point charge and the charge on the shell is treated as a smeared charge in accord with chemical intuition. The exponent of the valance Slater $s$-orbital is optimized for all the atom types, except for bromine and iodine. For these atoms, a Slater $3 s$ orbital is optimized rather than the valence orbital (See THEORY). 
Table 4: The optimized exponent for the polarizable Gaussian and Slater $s$-type orbitals represented by $\beta$ and $\zeta$ in $\mathrm{nm}^{-1}$, rescpectively. Slater $3 \mathrm{~s}$ orbital has been optimized rather than the valence Slater $s$-orbital for Bromine and Iodine (See THEORY).

\begin{tabular}{lcc}
\hline Polarizability Type & $\beta(\sigma)$ & $\zeta(\sigma)$ \\
\hline ha & $12.05(0.04)$ & $21.39(0.08)$ \\
hp & $11.81(0.08)$ & $22.03(0.33)$ \\
hw & $11.08(0.01)$ & $16.81(0.01)$ \\
c1 & $9.43(0.22)$ & $23.34(0.41)$ \\
c2 & $9.74(0.05)$ & $23.68(0.17)$ \\
ca & $9.58(0.03)$ & $23.36(0.06)$ \\
c3 & $12.12(0.01)$ & $32.88(0.01)$ \\
n & $10.18(0.11)$ & $24.88(0.28)$ \\
na & $11.25(0.89)$ & $24.39(0.41)$ \\
no & $9.28(0.13)$ & $22.82(0.54)$ \\
o & $11.30(0.11)$ & $28.86(0.50)$ \\
ow & $19.31(0.16)$ & $42.28(0.01)$ \\
f & $11.75(0.07)$ & $29.28(0.33)$ \\
p2 & $7.60(0.08)$ & $20.64(0.02)$ \\
p4 & $7.45(0.41)$ & $20.64(0.21)$ \\
s & $7.80(0.09)$ & $23.77(0.28)$ \\
cl & $9.70(0.17)$ & $27.44(0.19)$ \\
br & $8.96(1.85)$ & $22.11(0.76)$ \\
i & $7.68(0.78)$ & $22.88(0.39)$ \\
\hline
\end{tabular}

\section{Atomic Charges}

Fig. 3 displays histograms of the atomic partial charges generated using electrostatic potential fitting for 2070 molecules from the Alexandria library. The generated charges are chemically intuitive for most of the atom types. For instances, the charges assigned to oxygen are negative in most molecules, except for special cases such as (the positive) nitronium ion, nitrosyl bromide, $\mathrm{N}$ oxonitramide, where oxygen has a small positive charge. However, the algorithm often generates a negative charge for hydrogens that are connected to aliphatic carbons, denoted by ha atom type in Fig. 3 and the distribution for carbon atoms are unreasonably wide as well. This is indeed a known problem of the ESP-fitting algorithm in general which leads to buried atoms, away from the ESP grid points, becoming a sync for the fitting algorithm, since they contribute little to the ESP. ${ }^{32}$ 


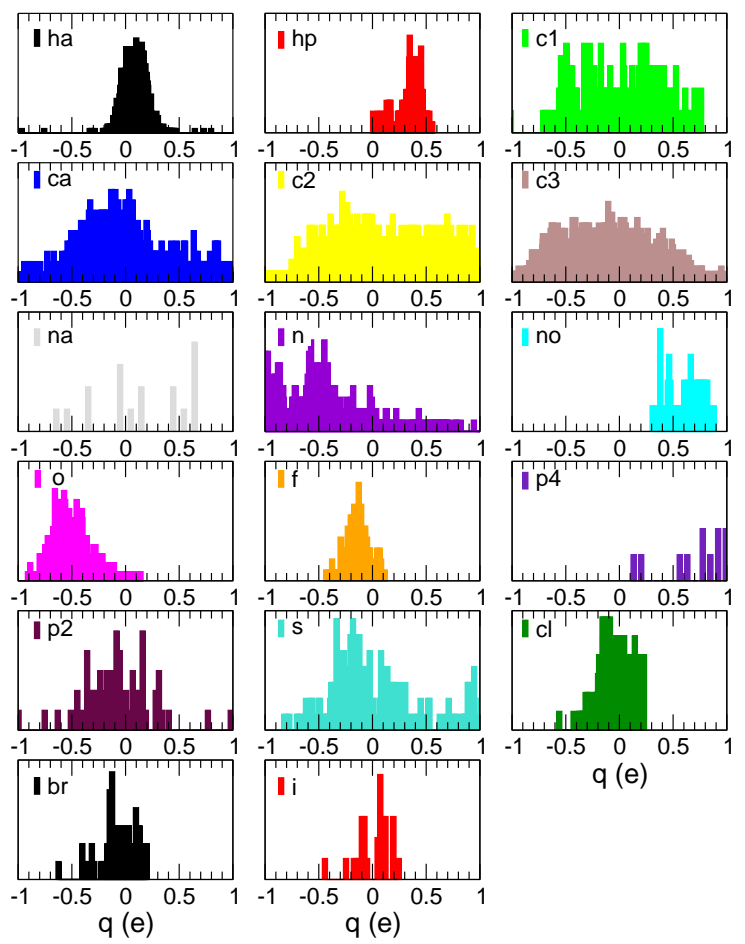

Figure 3: Histogram of atomic partial charges.

\section{Molecular Polarizability}

The obtained atomic polarizabilities were evaluated by computing molecular isotropic polarizabilities using Eqn. 11 (Fig. 4A). Table 5 shows that the additive approach used in Alexandria results in slightly lower RMSD than B3LYP/aug-cc-pVTZ. This can be explained, in part, by the fact that B3LYP/aug-cc-pVTZ overestimates the dipole polarizability of conjugated molecules such as tetracene, phenazine, and chrysene due to the presence of delocalized $\pi$-electron clouds. The diagnosis of the polarizability of delocalized electron densities is beyond the scope of this paper and has been discussed in detail elsewhere. ${ }^{53,55,89-91}$ The additive approach is ignorant of the electron delocalization due to being a simple linear fit, and thus, results in a lower RMSD $\left(0.30 \AA^{3}\right)$ than for B3LYP/aug-cc-PVTZ $\left(0.37 \AA^{3}\right)$. However, it cannot be determined how well the additive approach works for compounds outside the training set. The results also indicate that Alexandria has lower RMSD for the molecular isotropic polarizabilities than for Bosque et al. ${ }^{54}$ (RMSD $=0.58$ $\AA^{3}$ ) (Table 5). This can be explained by the fact the Alexandria has more polarizability types than 
elements.

The polarizability tensor was computed for all compounds using Eqn. 13 by applying electric fields with a strength of $10 \mathrm{Vnm}^{-1}$ in the $x, y$ and $z$ directions and optimizing the shell positions. The non-additive isotropic polarizability was then calculated using Eqn. 14 and compared to experimental data (Fig. 4B). The RMSD values obtained for the Alexandria charge models are very close to the RMSD value for B3LYP/aug-cc-pVTZ (Table 6). The MSE values show that the systematic errors correspond to less than $2 \%$ of the average $\alpha$. The diagonal elements of the polarizability tensor were compared to the B3LYP calculations (Table 7). Aromatic, conjugated, and annelated compounds were excluded from the statistics for reasons outlined above. The statistics show a good agreement between the Alexandria polarizable charge models and the B3LYP/aug-cc-pVTZ level of theory, even though the RMSD is slightly larger than for the isotropic (the average of the diagonal components) polarizability.

Table 5: Isotropic polarizability $\alpha\left(\AA^{3}\right)$ calculated using Eqn. 11 and quantum-mechanically (B3LYP/aug-cc-pVTZ). $N$ is the number of molecules. Reference is experimental data. ${ }^{92,93}$

\begin{tabular}{cllccc}
\hline Method & $N$ & slope & $R(\%)$ & RMSD & MSE \\
\hline DFT & 1168 & 1.025 & 99.70 & 0.37 & 0.04 \\
Alexandria & 1168 & 0.993 & 99.77 & 0.30 & 0.00 \\
Bosque & 1168 & 0.982 & 99.55 & 0.58 & -0.40 \\
\hline
\end{tabular}

Table 6: Isotropic polarizability $\alpha\left(\AA^{3}\right)$ calculated using Eqn. 14 and quantum-mechanically (B3LYP/aug-cc-pVTZ). $\mathrm{N}$ is the number of compounds. Reference is experimental data. ${ }^{92,93}$

\begin{tabular}{rccccc}
\hline Method & $\mathrm{N}$ & Slope & $R(\%)$ & RMSD & MSE \\
\hline DFT & 1150 & 1.01 & 99.70 & 0.36 & 0.05 \\
PPC & 1150 & 1.03 & 99.80 & 0.46 & 0.28 \\
PGC & 1150 & 1.02 & 99.80 & 0.46 & 0.28 \\
PSC & 1150 & 1.02 & 99.80 & 0.45 & 0.27 \\
\hline
\end{tabular}




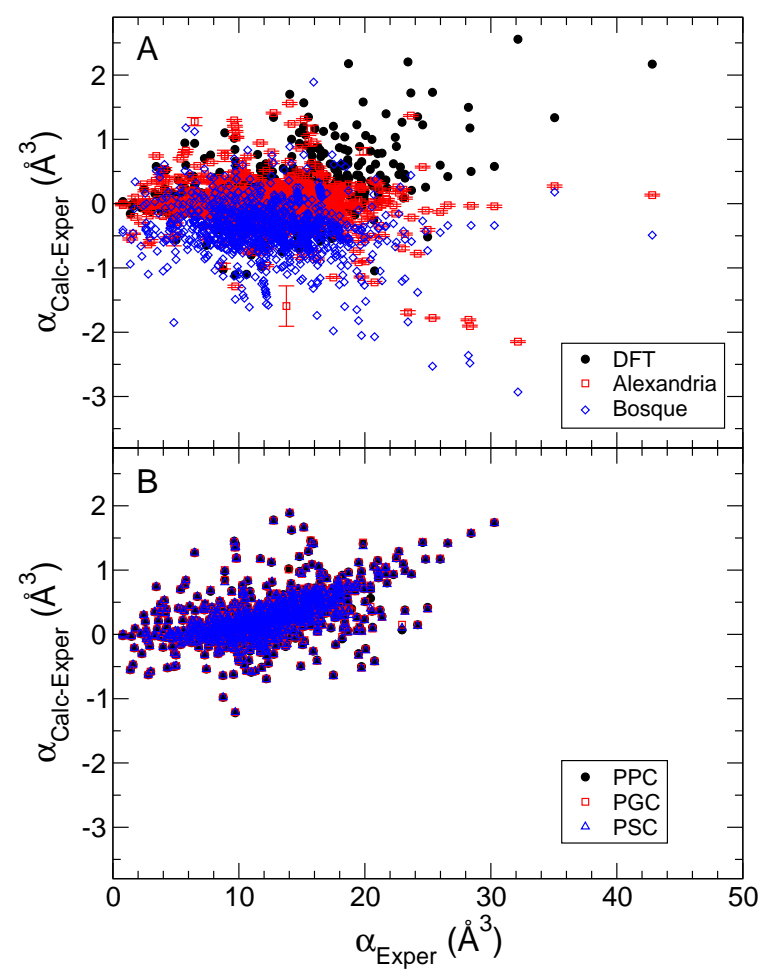

Figure 4: Residual plot for the molecular isotropic polarizability $\left(\AA^{3}\right)$ calculated A) using DFT (B3LYP/aug-cc-pVTZ) and by Eqn. 11 and B) calculated by Eqn. 14 based on Alexandria charge models.

Table 7: Diagonal components of the polarizability tensor $\alpha\left(\AA^{3}\right)$. N is the number of compounds. Reference is the B3LYP/aug-cc-pVTZ level of theory.

\begin{tabular}{cccccc}
\hline Method & $\mathrm{N}$ & Slope & $R(\%)$ & RMSD & MSE \\
\hline PPC & 1349 & 1.01 & 96.57 & 1.35 & 0.12 \\
PGC & 1349 & 1.01 & 96.60 & 1.36 & 0.14 \\
PSC & 1349 & 1.01 & 96.57 & 1.37 & 0.12 \\
\hline
\end{tabular}




\section{Dipole and Quadrupole Moments of Isolated Molecules}

Table 8 compares the predicted total dipole moment to experimental data for rigid molecules only, as the impact of the vibrational averaging over the accessible conformations of flexible molecules is expected to be significant. "Rigid molecules" were those determined to have no rotatable bonds. The RMSD and MSE values indicate that the predicted accuracy for total dipole moments by Alexandria polarizable charges is the same as that for B3LYP/aug-cc-pVTZ level of theory. The scattering of residuals obtained from this comparison is homogenous, indicating that the error in predicting the total dipole moment is not systematic (Fig. 5). Due to the lack of experimental data, the accuracy of the Alexandria charge models in computing the components of the dipole vector and the diagonal elements of the quadruple tensor was evaluated by comparing to the B3LYP calculations (Table 9). Here, the comparison is carried out for all molecules, both rigid and flexible. The results display that the dipole components along the $x, y$ and $z$ directions predicted by Alexandria charge models are in excellent agreement with the B3LYP calculations. The quadrupole tensor components (Table 10), on the other hand, are underestimated in comparison to DFT, while the RMSD values are consistent with previous studies. ${ }^{94}$

Table 8: Total dipole moment $\mu$ (Debye). $\mathrm{N}$ is the number of compounds. Reference is experimental data. ${ }^{92,93}$

\begin{tabular}{rccccc}
\hline Method & $\mathrm{N}$ & Slope & $R(\%)$ & RMSD & MSE \\
\hline DFT & 478 & 1.05 & 98.10 & 0.30 & 0.08 \\
PPC & 478 & 1.05 & 98.10 & 0.31 & 0.10 \\
PGC & 478 & 1.04 & 97.70 & 0.32 & 0.09 \\
PSC & 478 & 1.04 & 98.00 & 0.31 & 0.08 \\
\hline
\end{tabular}

Table 9: Components of the dipole vector $\boldsymbol{\mu}$ (Debye). $\mathrm{N}$ is the number of compounds. Reference is the B3LYP/aug-cc-pVTZ level of theory.

\begin{tabular}{cccccc}
\hline Method & $\mathrm{N}$ & Slope & $R(\%)$ & RMSD & MSE \\
\hline PPC & 1349 & 0.98 & 99.70 & 0.16 & 0.00 \\
PGC & 1349 & 0.98 & 99.70 & 0.15 & 0.00 \\
PSC & 1349 & 0.98 & 99.67 & 0.17 & 0.00 \\
\hline
\end{tabular}


Table 10: Diagonal components of the quadrupole tensor $\boldsymbol{\theta}$ (Buckingham). $\mathrm{N}$ is the number of compounds. Reference is the B3LYP/aug-cc-pVTZ level of theory.

\begin{tabular}{cccccc}
\hline Method & $\mathrm{N}$ & Slope & $R(\%)$ & RMSD & MSE \\
\hline PPC & 1349 & 1.55 & 96.73 & 2.23 & -0.05 \\
PGC & 1349 & 1.55 & 96.53 & 2.25 & -0.06 \\
PSC & 1349 & 1.55 & 95.77 & 2.32 & -0.06 \\
\hline
\end{tabular}

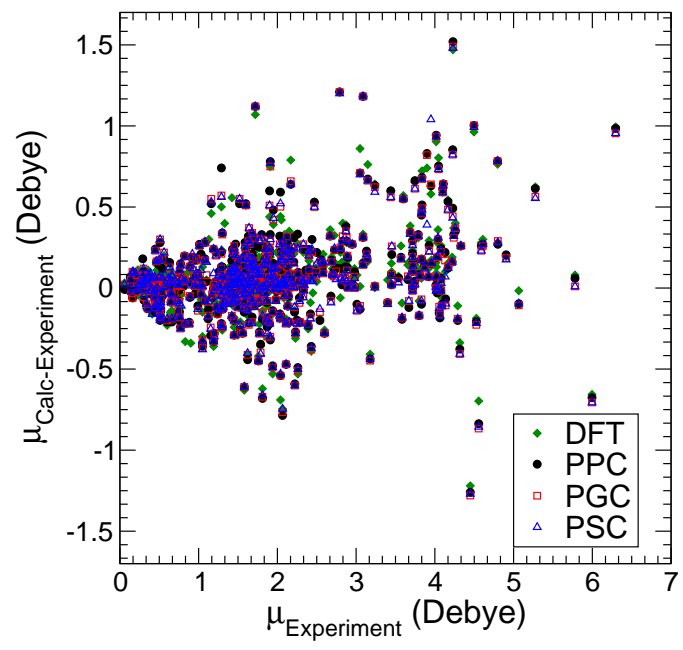

Figure 5: Residual plot of total molecular dipole moment (Debye) from DFT (B3LYP/aug-ccpVTZ) and Alexandria charge models. 


\section{Dipole Moment of Dimers and Clusters}

The Alexandria polarizable charge models were validated by computing the components of the dipole vector for different sets of homo- and hetero-dimers of common organic molecules and water clusters up to 10 monomers (Table 1). We also carried out the same calculations with fixed point charges without polarization (derived from an ESP fit and denoted here by PC) because this is widely used in empirical force fields such as GAFF, ${ }^{72}$ OPLS (Optimized Potential for Liquid Simulations), ${ }^{95}$ and CGenFF (CHARMM General Force Field). ${ }^{96}$

The total RMSD from DFT dipole moments decreases going from the fixed point charge model to polarizable charges by more than $50 \%$ (Table 11). The scattering of the residuals indicates that the error is random for the polarizable charges, while it is systematic for the fixed point charge (Fig. 6).

The results show that the polarizable point charge is as accurate as the polarizable smeared charges in predicting the dipole moments of the dimers at the equilibrium geometry. For the water clusters, the RMSD was found to be 0.07 and 0.04 (Debye) for the PC and PPC models respectively. In contrast, for popular fixed-charge models such as $\mathrm{TIP} 3 \mathrm{P}^{97}$ or $\mathrm{SPC} / \mathrm{E},{ }^{98}$ which have monomer dipoles of the order of 2.3 Debye instead of the experimental 1.85 Debye, a dimer dipole moment of 3.8 (Debye) is found for the water dimer, ${ }^{4,84}$ which deviates significantly from 2.4 to 2.7 (Debye) measured experimentally. ${ }^{99}$ Fig. 7 shows the total dipole moment of a water dimer at different separations along the hydrogen bond-breaking coordinates. Both PC and PPC fail to reproduce the dipole moment at short distances, while PGC and PSC are in good agreement with the B3LYP/augcc-pVTZ level of theory. The RMSD averaged over 191 conformations of the water dimer is 0.40 for PC, 0.24 for PPC, and 0.04 (Debye) for both PGC and PSC. However, further evaluations of other properties of water clusters, e.g. the vibrational spectra ${ }^{100}$ and exchange repulsion ${ }^{101}$ would be needed to scrutinize the effect of the water atomic charge distributions more rigorously.

The performance of the Alexandria charge models was also compared for different non-covalent interactions stabilizing homo- and hetero-dimer of organic compounds (Table 12). Consistently, the polarizable charges displayed a better performance than the PC. The results also exhibit that the 
RMSD value obtained for the halogen bond and halogen- $\pi$ interactions are higher than the other types of interactions for all the charge models compared in Table 12.

Table 11: Root-mean square deviation for Alexandria charge models from QM dipole vector $\boldsymbol{\mu}$ components (Debye) at the B3LYP/aug-cc-pVTZ level of theory.

\begin{tabular}{lccccc}
\hline Model & S66 & X40 & SHBC & Water & Total \\
\hline PC & 0.21 & 0.29 & 0.18 & 0.07 & 0.20 \\
PPC & 0.05 & 0.17 & 0.12 & 0.04 & 0.08 \\
PGC & 0.06 & 0.17 & 0.11 & 0.05 & 0.09 \\
PSC & 0.06 & 0.16 & 0.11 & 0.06 & 0.09 \\
\hline
\end{tabular}

Table 12: Root-mean square deviation for Alexandria charge models from QM dipole vector $\boldsymbol{\mu}$ components (Debye) at the B3LYP/aug-cc-pVTZ level of theory for specific interactions. $\mathrm{N}$ is the number of dimers

\begin{tabular}{llcccc}
\hline Interaction & $\mathrm{N}$ & PC & PPC & PGC & PSC \\
\hline$\pi-\pi$ & 6 & 0.10 & 0.01 & 0.03 & 0.02 \\
Halogen- $\pi$ & 4 & 0.41 & 0.24 & 0.25 & 0.23 \\
OH- $\pi$ & 4 & 0.16 & 0.12 & 0.11 & 0.11 \\
Halogen bond & 20 & 0.23 & 0.29 & 0.20 & 0.23 \\
Hydrogen bond & 10 & 0.49 & 0.12 & 0.12 & 0.11 \\
Dispersion & 4 & 0.15 & 0.13 & 0.13 & 0.13 \\
Induction & 4 & 0.06 & 0.05 & 0.05 & 0.05 \\
T-Shaped Aromatic Dimers & 4 & 0.26 & 0.05 & 0.01 & 0.03 \\
\hline
\end{tabular}




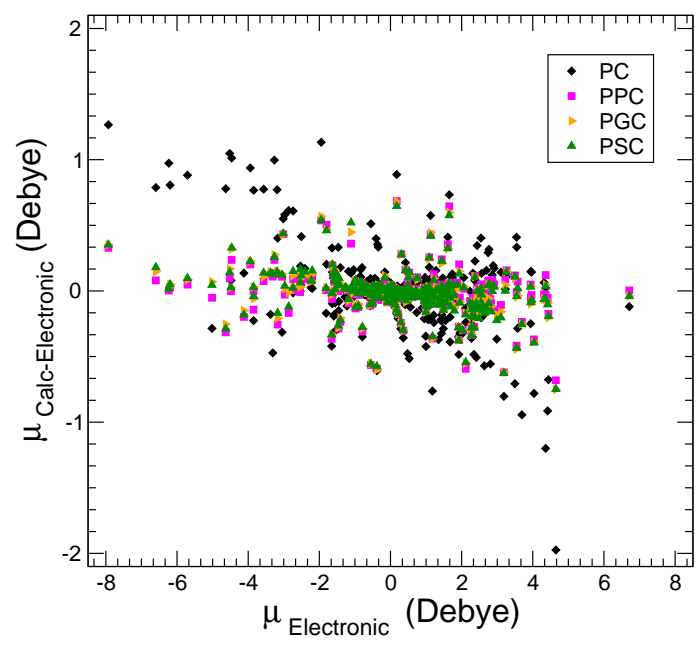

Figure 6: Residual plot of the components of the dipole vector obtained for homo- and heterodimers and water clusters using Alexandria charge models.

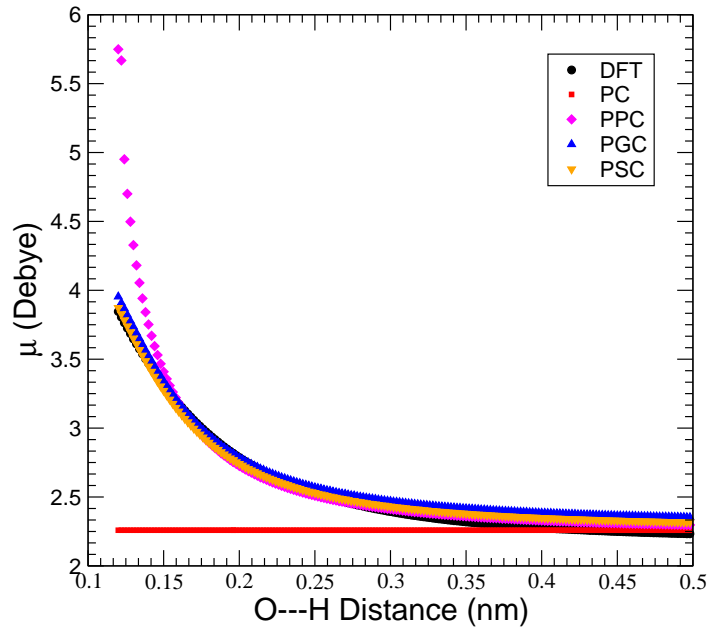

Figure 7: Total dipole moment (Debye) for water dimer. 


\section{Electrostatic Interaction Energy of Water Dimer}

Fig. 8 plots the electrostatic component of the interaction energy $\left(E^{e l e c}\right)$ for the water dimer along the hydrogen bond-breaking coordinate using SAPT2+/aug-cc-pVTZ and the Alexandria charge models. The $\mathrm{O} \cdots \mathrm{H}$ distance is varied from 0.12 to $0.5 \mathrm{~nm}$. Our quantum calculations find $E^{e l e c}$ to be $-33.11 \mathrm{~kJ} / \mathrm{mol}$ at the equilibrium distance $(0.196 \mathrm{~nm})$ in the aug-cc-pVTZ basis, which is consistent with $-30.25,-34.01$ and $-35.18(\mathrm{~kJ} / \mathrm{mol})$ reported in other studies at different high levels of theory. ${ }^{12,102,103}$ The variation between these values somehow reflects the uncertainty in quantum chemistry methods in computing intermolecular interaction energies due to different levels of theory. We find $E^{e l e c}$ to be -17.93 for PC, -25.06 for PPC, -31.65 for PGC, and $-32.58(\mathrm{~kJ} / \mathrm{mol})$ for PSC at the equilibrium geometry. The RMSD averaged over 191 conformations of the water dimer is 24.62 for PC, 18.15 for PPC, 1.93 for PGC, and $1.60(\mathrm{~kJ} / \mathrm{mol})$ for PSC. This shows that the deviation from the SAPT2+ electrostatic interaction energy systematically decreases with increasingly realistic charge models, in agreement with previous studies. ${ }^{12,94}$

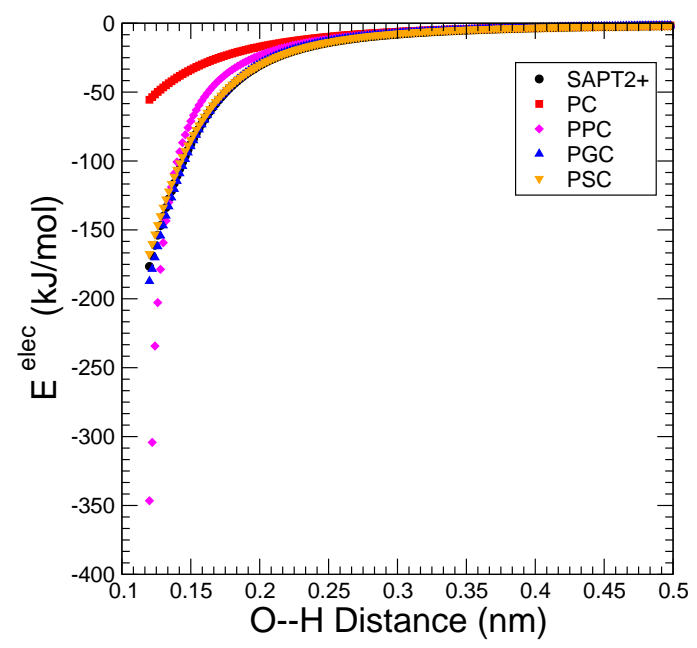

Figure 8: Electrostatic interaction energy $(\mathrm{kJ} / \mathrm{mol})$ for water dimer. 


\section{DISCUSSION}

The importance of using polarization and damped (smeared) Coulomb interactions in molecular mechanics force fields has been pointed out in a number of previous studies. ${ }^{2,8,9,12,17,19}$ However, modeling of molecular polarizability requires a description of the atomic polarizability, which cannot be determined experimentally. ${ }^{50}$ Using smeared charge models such as Gaussian and Slater $s$-type atomic orbitals also require determining the exponents of the orbitals. As a result, atomic polarizabilities and orbital exponents expand the parameter space of empirical force fields somewhat, by two parameters per atom. In order for force fields to be transferable it is therefore important to validate that these parameters can be determined by the atom type alone, which is what we attempt in this paper. It is possible that the introduction of more accurate physics in the molecular models will reduce the need for having so many atom types as if common in most force fields, but this remains to be determined.

The most widely used Drude polarizable force field is likely polarizable CHARMM. ${ }^{6,42,104}$ It treats atomic partial charges as point charges. The sign of the charge is chosen to be positive on the core-representing the nucleus — and negative on the Drude-representing the electron cloud - to be intuitively consistent. In order to reduce the number of shell particles and to avoid the polarization catastrophe, polarizable CHARMM assigns polarizability only to non-hydrogen atoms and damps the electrostatic 1-2 and 1-3 pair interactions. ${ }^{6,42,104}$ To compensate for this, polarizabilities are scaled down by $15 \%,{ }^{42}$ but in addition anisotropic polarizabilities are used for oxygen atoms in e.g. carbonyl groups, ${ }^{104}$ as introduced for water early on. ${ }^{4}$ It has been shown though, that assigning polarizability to hydrogens improves the description of the water polarization and allows to use the full molecular polarizability rather than scaling it down. ${ }^{105}$ Moreover, the atomic polarizability of 0.432 and $0.384 \AA^{3}$ obtained in this study for polar and non polar hydrogens, respectively, suggest that hydrogens contribute significantly to the molecular polarizability. The AMOEBA force field assigns a polarizability of $0.496 \AA^{3}$ to hydrogens ${ }^{7}$ which is similar to our values. Drude polarizable Gaussian charges have been developed for specific organic molecules such as carbon dioxide $^{20}$ and water. ${ }^{24}$ In these models, some shells have a positive charge and some others have 
a negative charge and all the cores are neutral, which is counter-intuitive. Rappé and Goddard applied the fluctuating charge method to describe polarizability of Slater charge densities. ${ }^{15}$ The major problem with the fluctuating charge method is that it does not describe the out-of-plane polarization. ${ }^{1}$ To circumvent this shortcoming, Donchev et al. parametrized a Drude model with Slater charges on a small set of monomers and dimers. ${ }^{94}$ Other Slater charge models are, to our knowledge, non-polarizable. ${ }^{2,12,17,19,29}$ Therefore, the main goal in this paper was to build a Drude model with $s$-type Gaussian and Slater density functions. We attempted to provide a general and robust platform to determine the values of atomic polarizabilities and to optimize the exponent of $1 s$-Gaussian and $n s$-Slater $(n=1,2,3)$ orbitals for $\mathrm{H}, \mathrm{C}, \mathrm{N}, \mathrm{O}, \mathrm{P}, \mathrm{S}$ and halogens $(\mathrm{F}, \mathrm{Cl}, \mathrm{Br}, \mathrm{I})$ to be transferable among organic molecules.

Analysis of the polarizabilities, dipoles, and quadrupoles obtained for the large number of molecules used in this study strongly suggests that the atomic polarizabilities and the exponents of the spherical Gaussian and Slater density functions are transferable among the molecules used and are likely to be transferable beyond the Alexandria library. We found that both the additive (Eqn. 11) and the non-additive (Eqn. 14) approaches to calculating the molecular isotropic polarizability yielded results in agreement with the reference data for the molecules used. This conclusion, however, is not entirely general because the non-additive effects of conjugated chains with multiple double bonds was not addressed in this work, mainly due to systematic errors in the applied density functional theory method. ${ }^{55}$ A recently developed approach to decompose the linear response properties into additive and cooperative contributions can be used to quantify the non-additive effects in the polarizability of conjugated chains. ${ }^{106}$

Evaluation of the charge models on dimers and complexes showcases the poor performance of the point charge model in predicting the dipole vector of the homo- and hetero-dimer of organic molecules. The explicit inclusion of the charge polarization decreased the RMSD from the B3LYP/aug-cc-pVTZ calculations by 50\% (Table 11). A similar improvement can be anticipated for simulating the liquid phase, however, the error may accumulate and/or cancel accross multiple interacting dimers in the liquid phase. Polarizable charges significantly decreased the deviation 
from the components of the dipole vector calculated at the B3LYP/aug-cc-pVTZ level of theory for dimers stabilized by hydrogen bonding and $\pi-\pi$ stacking, which are the main non-covalent interactions stabilizing secondary and tertiary structures of nucleic acids. However, in the case of halogen bonding, the RMSD is relatively high even though the RMSD obtained for the polarizable charges was much smaller than the fixed point charge (Table 12). Halogen bonding is important in molecular recognition, because about half of the drugs or drug-like compounds contain fluorine and chlorine. ${ }^{107,108}$ Therefore, the presented polarizable models need to be improved further to describe the " $\sigma$ hole" in halogen bonds. One possible solution is to go beyond atomic centers through off-center virtual sites. ${ }^{104}$ Among the widely used force fields, OPLS was first to explicitly treat the " $\sigma$ hole" in halogen bonds by defining a massless virtual site (X-site) carrying a positive charge on $\mathrm{Cl}, \mathrm{Br}$, and I. ${ }^{109}$ Similar extensions were introduced into the CHARMM Generalized Force Field ${ }^{110}$ and the Polarizable CHARMM force field. ${ }^{85}$

We found that the polarizable point charge is as accurate as the polarizable Gaussian and Slater charges for reproducing the dipole vector and the diagonal elements of the polarizability- and quadrupole tensors of the isolated molecules in the gas phase. This is consistent with the fact that the interactions at short intramolecular distances are excluded in our force field calculations to attenuate the quantum mechanical effect. In order to study the charge penetration effect at short distances, the electrostatic interaction energy and the total dipole moment were calculated for 191 conformations of a water dimer at separation distances from 0.12 to $0.5 \mathrm{~nm}$. Consistent with previous studies, ${ }^{12,17}$ we find that the polarizable point charge fails to describe both the dipole moment (Fig. 7) and the electrostatic energy (Fig. 8) at very short distances. The results also showed that, although all the polarizable charges accurately predicted the dipole moment of the water dimer at equilibrium geometry, the PPC model deviated from the reference electrostatic energy of the equilibrium geometry by as much as $8.05(\mathrm{~kJ} / \mathrm{mol})$, while the deviation was 1.46 $(\mathrm{kJ} / \mathrm{mol})$ for PGC and $0.53(\mathrm{~kJ} / \mathrm{mol})$ for PSC. This suggests that the charge penetration effect is needed in addition to the charge polarization to yield accurate electrostatic energies even at the equilibrium distance. 
In summary, the atomic polarizabilities and the exponents of the $s$-type Gaussian and Slater density functions presented in this work reproduce electrostatic properties for molecules of the Alexandria library and are likely transferable to other compounds with similar chemistries. Although, the Alexandria polarizable point charge is accurate enough to be used for prediction of the isotropic polarizability and dipole moments of organic molecules in the gas phase, this work strongly suggests that the atomic partial charges in molecular dynamics simulations should be treated as smeared charges to produce accurate electrostatic energies, particularly when simulating the condensed phase. 


\section{Acknowledgments}

The Swedish research council is acknowledged for financial support to DvdS (grant 2013-5947) and for a grant of computer time (SNIC2016/34-44) through the High Performance Computing Center North in Umeå, Sweden. The Helmholtz Association through the Center for Free-Electron Laser Science at DESY is acknowledged as well.

\section{Graphical TOC Entry}

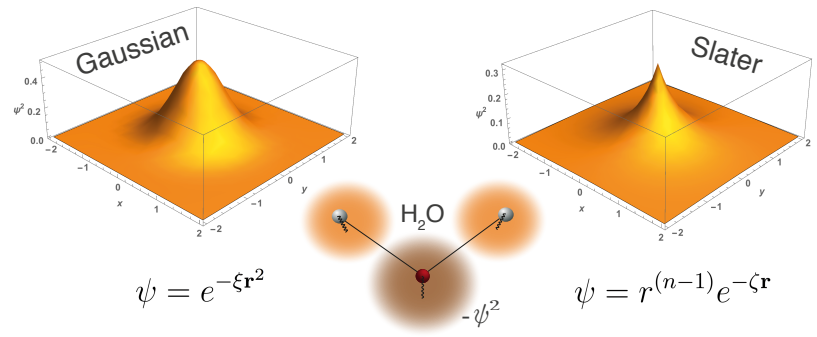

\section{References}

(1) Cieplak, P.; Dupradeau, F.-Y.; Duan, Y.; Wang, J. J. Phys. Condens. Matter 2009, 21, 333102.

(2) Wang, B.; Truhlar, D. G. J. Chem. Theory Comput. 2010, 6, 3330-3342.

(3) Jordan, P. C.; van Maaren, P. J.; Mavri, J.; van der Spoel, D.; Berendsen, H. J. C. J. Chem. Phys. 1995, 103, 2272-2285.

(4) van Maaren, P. J.; van der Spoel, D. J. Phys. Chem. B. 2001, 105, 2618-2626.

(5) Ren, P.; Ponder, J. W. J. Comput. Chem. 2002, 23, 1497-1506.

(6) Anisimov, V. M.; Lamoureux, G.; Vorobyov, I. V.; Huang, N.; Roux, B.; MacKerell, Jr., A. D. J. Chem. Theory Comput. 2005, 1, 153-168. 
(7) Ponder, J. W.; Wu, C.; Ren, P.; Pande, V. S.; Chodera, J. D.; Schnieders, M. J.; Haque, I.; Mobley, D. L.; Lambrecht, D. S.; DiStasio Jr., R. A.; Head-Gordon, M.; Clark, G. N. I.; Johnson, M. E.; Head-Gordon, T. J. Phys. Chem. B 2010, 114, 2549-2564.

(8) Ahlström, P.; Wallqvist, A.; Engström, S.; Jönsson, B. Mol. Phys. 1989, 68, 563-581.

(9) Thole, B. T. Chem. Phys. 1981, 59, 341-345.

(10) Lindan, P. J. D. Mol Simul 1995, 14, 303-312.

(11) Allen, M. P.; Tildesley, D. J. Computer Simulation of Liquids, 2nd ed.; Oxford Science Publications: Oxford, 2017.

(12) Wang, Q.; Rackers, J. A.; He, C.; Qi, R.; Narth, C.; Lagardere, L.; Gresh, N.; Ponder, J. W.; Piquemal, J.-P.; Ren, P. J. Chem. Theory Comput. 2015, 11, 2609-2618.

(13) Hall, G. G.; Tsujinaga, K. Theor. Chim. Acta. 1986, 69, 425-436.

(14) Hall, G. G.; Smith, C. M. Theor. Chim. Acta. 1986, 69, 71-81.

(15) Rappé, A. K.; Goddard III, W. A. J. Phys. Chem. 1991, 95, 3358-3363.

(16) Kiss, P. T.; Sega, M.; Baranyai, A. J. Chem. Theory Comput. 2014, 10, 5513-5519.

(17) Öhrn, A.; Hermida-Ramon, J. M.; Karlström, G. J. Chem. Theory Comput. 2016, 12, 22982311.

(18) Wang, F.-F.; Kumar, R.; Jordan, K. D. Theor. Chem. Acc. 2012, 131, 1132.

(19) Wang, B.; Truhlar, D. G. J. Chem. Theory Comput. 2014, 10, 4480-4487.

(20) Jiang, H.; Moultos, O. A.; Economou, I. G.; Panagiotopoulos, A. Z. J. Phys. Chem. B 2016, 120, 984-994.

(21) Kiss, P. T.; Baranyai, A. J. Chem. Phys. 2014, 141, 114501. 
(22) Baranyai, A.; Kiss, P. T. J. Chem. Phys. 2010, 133, 144109.

(23) Baranyai, A.; Kiss, P. T. J. Chem. Phys. 2011, 135, 234110.

(24) Kiss, P. T.; Baranyai, A. J. Chem. Phys. 2012, 137, 84506-84508.

(25) Elking, D.; Darden, T.; Woods, R. J. J. Comput. Chem. 2007, 28, 1261-1274.

(26) Ghahremanpour, M. M.; van Maaren, P.; van der Spoel, D. Alexandria Library [Data set]. Zenodo. 2017; http: / / doi.org/10.5281/zenodo.1004711.

(27) Ghahremanpour, M. M.; van Maaren, P. J.; van der Spoel, D. Sci. Data 2018, 5, 180062.

(28) Cailliez, F.; Pernot, P. J. Chem. Phys. 2011, 134, 054124.

(29) Verstraelen, T.; Vandenbrande, S.; Heidar-Zadeh, F.; Vanduyfhuys, L.; Van Speybroeck, V.; Waroquier, M.; Ayers, P. W. J. Chem. Theory Comput. 2016, 12, 3894-3912.

(30) Murray, J. S.; Politzer, P. Wiley Interdiscip. Rev. Comput. Mol. Sci. 2011, 1, 153-163.

(31) Besler, B. H.; Merz Jr., K. M.; Kollman, P. A. J. Comput. Chem. 1990, 11, 431-439.

(32) Sigfridsson, E.; Ryde, U. J. Comput. Chem. 1998, 19, 377-395.

(33) Rick, S. W.; Stuart, S. J.; Berne, B. J. J. Chem. Phys. 1994, 101, 6141-6156.

(34) Hentschke, R.; Aydt, E. M.; Fodi, B.; Schöckelmann, E. Molekulares Modellieren mit Kraftfeldern; Bergische Universität Wuppertal: Wuppertal, Germany, 2004.

(35) Guseinov, I. I. J. Phys. B: Atom. Molec. Phys. 1970, 3, 1399.

(36) Elking, D. M.; Cisneros, G. A.; Piquemal, J.-P.; Darden, T. A.; Pedersen, L. G. J. Chem. Theory Comput. 2010, 6, 190-202.

(37) Dick, B. G.; Overhauser, A. W. Phys. Rev. 1958, 112, 90-103. 
(38) Saint-Martin, H.; Hernández-Cobos, J.; Bernal-Uruchurtu, M. I.; Ortega-Blake, I.; Berendsen, H. J. C. J. Chem. Phys. 2000, 113, 10899-10912.

(39) Lamoureux, G.; Roux, B. J. Phys. Chem. 2003, 119, 3025-3039.

(40) Geerke, D. P.; van Gunsteren, W. F. J. Chem. Theory Comput. 2007, 3, 2128-2137.

(41) Lopes, P. E. M.; Lamoureux, G.; Roux, B.; MacKerell, Jr., A. D. J. Phys. Chem. B. 2007, 111,1 .

(42) Lopes, P. E. M.; Huang, J.; Shim, J.; Luo, Y.; Li, H.; Roux, B.; MacKerell, J., Alexander D. J. Chem. Theory Comput 2013, 9, 5430-5449.

(43) Lemkul, J.; Roux, B.; van der Spoel, D.; MacKerell, A. J. Comput. Chem. 2015, 36, 14731479.

(44) Albaugh, A.; Head-Gordon, T. J. Chem. Theory Comput. 2017, 13, 5207-5216.

(45) Huang, J.; Simmonett, A. C.; Pickard IV, F. C.; MacKerell Jr., A. D.; Brooks, B. R. J. Chem. Phys. 2017, 147, 161702.

(46) Li, A.; Voronin, A.; Fenley, A. T.; Gilson, M. K. J. Phys. Chem. B 2016, 120, 8668-8684.

(47) Chialvo, A. A.; Moucka, F.; Vlcek, L.; Nezbeda, I. J. Phys. Chem. B 2015, 119, 5010-5019.

(48) Lindan, P. J. D.; Gillan, M. J. J. Phys.: Condens. Matter 1993, 5, 1019-1030.

(49) Lemkul, J. A.; Huang, J.; Roux, B.; MacKerell, A. D. Chem. Rev. 2016, 116, 4983-5013.

(50) Silberstein, L. Philos. Mag. 1917, 33, 92-128.

(51) Kang, Y. K.; Jhon, M. S. Theor. Chem. Acc. 1982, 61, 41-48.

(52) McMeekin, T. L.; Groves, M. L.; Wilensky, M. Biochem. Biophys. Res. Commun. 1962, 7, $151-160$. 
(53) Alparone, A. Chem. Phys. 2013, 410, 90 - 98.

(54) Bosque, R.; Sales, J. J. Chem. Inf. Comput. Sci. 2002, 42, 1154-1163.

(55) Limacher, P. A.; Mikkelsen, K. V.; Lüthi, H. P. J. Chem. Phys. 2009, 130, 194114.

(56) Alipour, M. J. Phys. Chem. A 2014, 118, 5333-5342.

(57) Ghahremanpour, M. M.; van Maaren, P. J.; Ditz, J.; Lindh, R.; van der Spoel, D. J. Chem. Phys. 2016, 145, 114305.

(58) Hohenberg, P.; Kohn, W. Phys. Rev. 1964, 136, B864-B871.

(59) Becke, A. D. Phys. Rev. A 1988, 38, 3098-3100.

(60) Lee, C.; Yang, W.; Parr, R. G. Phys. Rev. B 1988, 37, 785-789.

(61) Becke, A. D. J. Chem. Phys. 1993, 98, 5648-5652.

(62) Kendall, R. A.; Dunning, Jr., T. H.; Harrison, R. J. J. Chem. Phys. 1992, 96, 6796-6806.

(63) Woon, D. E.; Dunning, Jr., T. H. J. Chem. Phys. 1993, 99, 1914-1929.

(64) Woon, D. E.; Dunning, Jr., T. H. J. Chem. Phys. 1993, 98, 1358-1371.

(65) ̆̌ezáč, J.; Riley, K. E.; Hobza, P. J. Chem. Theory Comput. 2011, 7, 2427-2438.

(66) ̆ Rezáč, J.; Riley, K. E.; Hobza, P. J. Chem. Theory Comput. 2014, 10, 1359-1360.

(67) ̆ezáč, J.; Riley, K. E.; Hobza, P. J. Chem. Theory Comput. 2012, 8, 4285-4292.

(68) Riley, K. E.; Hobza, P. J. Chem. Theory Comput. 2008, 4, 232-242.

(69) Temelso, B.; Archer, K. A.; Shields, G. C. J. Phys. Chem. A. 2011, 115, 12034-12046.

(70) O’Boyle, N. M.; Banck, M.; James, C. A.; Morley, C.; Vandermeersch, T.; Hutchison, G. R. J. Cheminf. 2011, 3, 33. 
(71) SMARTS - A Language for Describing Molecular Patterns. http://www.daylight.com/dayhtml/doc/theory/theory.smarts.html, 2008.

(72) Wang, J.; Wolf, R. M.; Caldwell, J. W.; Kollman, P. A.; Case, D. A. J. Comput. Chem. 2004, $25,1157-1174$.

(73) Case, D. et al. AMBER 2016. 2016; University of California, San Francisco.

(74) Bayly, C. I.; Cieplak, P.; Cornell, W. D.; Kollman, P. A. J. Phys. Chem. 1993, 97, 1026910280.

(75) Cerutti, D. S.; Swope, W. C.; Rice, J. E.; Case, D. A. J. Chem. Theory Comput. 2014, 10, 4515-4534.

(76) Pronk, S.; Páll, S.; Schulz, R.; Larsson, P.; Bjelkmar, P.; Apostolov, R.; Shirts, M. R.; Smith, J. C.; Kasson, P. M.; van der Spoel, D.; Hess, B.; Lindahl, E. Bioinformatics 2013, $29,845-54$.

(77) Freedman, D. A. Ann. Stat. 1981, 9, 1218-1228.

(78) Frederiksen, S. L.; Jacobsen, K. W.; Brown, K. S.; Sethna, J. P. Phys. Rev. Lett. 2004, 93, 165501.

(79) Mortensen, J. J.; Kaasbjerg, K.; Frederiksen, S. L.; Nørskov, J. K.; Sethna, J. P.; Jacobsen, K. W. Phys. Rev. Lett. 2005, 95, 216401.

(80) Frisch, M. J. et al. Gaussian 16 Revision A.03. 2016; Gaussian Inc. Wallingford CT.

(81) M., T. J. et al. Wiley Interdiscip. Rev. Comput. Mol. Sci. 2, 556-565.

(82) Jeziorski, B.; Moszynski, R.; Szalewicz, K. Chem. Rev. 1994, 94, 1887-1930.

(83) Parker, T. M.; Burns, L. A.; Parrish, R. M.; Ryno, A. G.; Sherrill, C. D. J. Chem. Phys. 2014, $140,094106$. 
(84) Yu, W.; Lopes, P. E. M.; Roux, B.; MacKerell, Jr., A. D. J. Chem. Phys. 2013, 138, 34508.

(85) Lin, F.-Y.; MacKerell, A. D. J. Chem. Theory Comput. 2018, 14, 1083-1098.

(86) Moelwyn-Hughes, E. A. Physical chemistry, 2nd ed.; Pergamon Press, New York, 1964.

(87) Miller, K. J. J. Am. Chem. Soc. 1990, 112, 8542-8553.

(88) Miller, K. J.; Savchik, J. A. J. Am. Chem. Soc. 1979, 101, 7206-7213.

(89) Rustagi, K.; Ducuing, J. Opt. Commun. 1974, 10, 258 - 261.

(90) Huzak, M.; Deleuze, M. S. J. Chem. Phys. 2013, 138, 024319.

(91) Champagne, B.; Perpête, E. A.; van Gisbergen, S. J. A.; Baerends, E.-J.; Snijders, J. G.; Soubra-Ghaoui, C.; Robins, K. A.; Kirtman, B. J. Chem. Phys. 1998, 109, 10489-10498.

(92) Lide, D. R. CRC Handbook of Chemistry and Physics 90th edition; CRC Press: Cleveland, Ohio, 2009.

(93) Rowley, R. L.; Wilding, W. V.; Oscarson, J. L.; Yang, Y.; Giles, N. F. Data Compilation of Pure Chemical Properties (Design Institute for Physical Properties; American Institute for Chemical Engineering: New York, 2012.

(94) Donchev, A. G.; Ozrin, V. D.; Subbotin, M. V.; Tarasov, O. V.; Tarasov, V. I. Proc. Natl. Acad. Sci. U.S.A. 2005, 102, 7829-7834.

(95) Harder, E. et al. J. Chem. Theory Comput. 2016, 12, 281-296.

(96) Vanommeslaeghe, K.; Hatcher, E.; Acharya, C.; Kundu, S.; Zhong, S. J. Comput. Chem. 2010, 31, 671-690.

(97) Jorgensen, W. L. J. Am. Chem. Soc. 1981, 103, 335-340.

(98) Berendsen, H. J. C.; Grigera, J. R.; Straatsma, T. P. J. Phys. Chem. 1987, 91, 6269-6271. 
(99) Gregory, J. K.; Clary, D. C.; Liu, K.; Brown, M. G.; Saykally, R. J. Science 1997, 275, 814-817.

(100) Jordan, K. D.; Sen, K. Chemical Modelling; The Royal Society of Chemistry, 2017; Vol. 13; pp 105-131.

(101) Kumar, R.; Wang, F.-F.; Jenness, G. R.; Jordan, K. D. J. Chem. Phys. 2010, 132, 014309.

(102) Glendening, E. D. J. Phys. Chem. A 2005, 109, 11936-11940.

(103) Su, P.; Li, H. J. Chem. Phys. 2009, 131, 014102.

(104) Harder, E.; Anisimov, V. M.; Vorobyov, I. V.; Lopes, P. E. M.; Noskov, S. Y.; MacKerell, A. D.; Roux, B. J. Chem. Theory Comput. 2006, 2, 1587-1597.

(105) Schropp, B.; Tavan, P. J. Phys. Chem. B 2008, 112, 6233-6240.

(106) Lambrecht, E., Daniel; Berquist 2018; https://doi .org/10.26434/chemrxiv . $5773968 \cdot \mathrm{V} 1$.

(107) Metrangolo, P.; Neukirch, H.; Pilati, T.; Resnati, G. Acc. Chem. Res. 2005, 38, 386-395.

(108) Lu, Y.; Liu, Y.; Xu, Z.; Li, H.; Liu, H.; Zhu, W. Exp. Op. Drug Disc. 2012, 7, 375-383.

(109) Jorgensen, W. L.; Schyman, P. J. Chem. Theory Comput. 2012, 8, 3895-3901.

(110) Soteras-Gutiérrez, I.; Lin, F. Y.; Vanommeslaeghe, K.; Lemkul, J. A.; Armacost, K. A.; Brooks III, C. L.; MacKerell Jr., A. D. Bioorg. Med. Chem. 2016, 24, 4812ấĽ̌ 4825. 